\title{
Snail precedes Slug in the genetic cascade required for the specification and migration of the Xenopus neural crest
}

\author{
Manuel J. Aybar ${ }^{1}$, M. Angela Nieto ${ }^{2}$ and Roberto Mayor ${ }^{1, *}$ \\ ${ }^{1}$ Millennium Nucleus in Developmental Biology, Facultad de Ciencias, Universidad de Chile, Casilla 653, Santiago, Chile \\ 2Instituto Cajal, CSIC, Doctor Arce 37, 28002, Madrid, Spain \\ *Author for correspondence (e-mail: rmayor@uchile.cl) \\ Accepted 23 October 2002
}

\section{SUMMARY}

The complex sequence of inductive events responsible for the generation of the neural crest at the border between the neural plate and the epidermis, triggers a genetic cascade involving several families of transcription factors. Two members of the Snail family, Snail and Slug, have both been implicated in this cascade. In chick and Xenopus, loss- and gain-of-function experiments have provided evidence that Slug plays a key role in neural crest development. However, in contrast to the chick, Snail rather than Slug is expressed in the premigratory neural crest in the mouse and, in Xenopus, Snail precedes Slug expression in this population. Thus, in order to study the function of Snail in neural crest development in Xenopus, we have carried out conditional gain- and loss-of-function experiments using different Snail constructs fused to a glucocorticoid receptor element.

We show that Snail is able to induce the expression of Slug and all other neural crest markers tested (Zic5, FoxD3, Twist and Ets1) at the time of specification. This activation is observed in whole embryos and in animal caps, in the absence of neural plate and mesodermal markers. We show that Snail is required for neural crest specification and migration and that it works as a transcriptional repressor. These functions have been previously attributed to Slug. However, Slug alone is unable to induce other neural crest markers in animal cap assays, and we show that Snail and Slug can be functionally equivalent when tested in overexpression studies. This suggests that, in Xenopus embryos, at least some of the functions previously attributed to Slug can be carried out by Snail. This is additionally supported by rescue experiments in embryos injected with dominant-negative constructs that indicate that Snail lies upstream of Slug in the genetic cascade leading to neural crest formation and that it plays a key role in crest development.

Key words: Snail, Slug, Neural crest, Crest specification, Crest migration, Zic5, FoxD3

\section{INTRODUCTION}

The neural crest comprises a unique set of cells, which segregates from the dorsal part of the neural tube. Prior to adopting their final fate, these cells migrate through the embryo, and generate a prodigious array of cell types, including neurones and support cells of the peripheral nervous system, pigment cells, smooth muscle, craniofacial cartilage, and bone and fin in amphibians and fish (for reviews, see LaBonne and Bronner-Fraser, 2000; Mayor and Aybar, 2001; Nieto, 2001). The neural crest is generated at the junction between the neural and nonneural ectoderm through a complex series of interactions, shortly after the induction of the nervous system (for reviews, see Knecht and Bronner-Fraser, 2002; Aybar and Mayor, 2002). In Xenopus and zebrafish, a gradient of BMP signalling together with posteriorising signals, such as Wnts, FGFs and retinoic acid have been implicated in this process (LaBonne and Bronner-Fraser, 1998; Marchant et al., 1998; Nguyen et al., 1998; Villanueva et al., 2002; GarcíaCastro et al., 2002). Once the neural crest has been induced at the border of the neural plate, the cells start to migrate along pathways dictated by their own internal programme and on signals received from the environment. The migration of the neural crest is known to follow the triggering of the epithelialmesenchymal transition (EMT), the process by which an epithelial cell converts to a mesenchymal cell, and delaminates from the epithelium (Hay, 1995; Duband et al., 1995; Perris and Perissinotto, 2000; Locascio and Nieto, 2001). Indeed, Snail family members have been implicated in triggering EMT, both in the formation of the neural crest and of the mesoderm during embryogenesis, and during tumour progression (for a review, see Nieto, 2002).

The function of one of the vertebrate Snail family members, Slug, has been studied in neural crest development by gain- and loss-of-function experiments in chick and Xenopus. In the chick embryo, antisense oligonucleotides directed against Slug mRNA can prevent neural crest migration (Nieto et al., 1994), whereas overexpression of Slug induces an increase in the production of the neural crest (Del Barrio and Nieto, 2002). Similarly, injections of Slug antisense RNA or of a dominant- 
negative form of Slug in Xenopus embryos reduces the expression of neural crest markers and inhibits the migration of the crest from the neural tube (Carl et al., 1999; LaBonne and Bronner-Fraser, 2000; Mayor et al., 2000). Conversely, overexpression of Slug led to an enlargement of the neural crest territory (LaBonne and Bronner-Fraser, 2000; Mayor et al., 2000). These results support the idea that Slug plays a key role in neural crest development in chick and amphibian embryos. However, mice homozygous for a null mutation in Slug are viable and display no obvious defects in neural crest formation, migration or development (Jiang et al., 1998). This can be explained by the fact that, in the mouse, Slug is not expressed in the premigratory neural crest but rather the other vertebrate family member, Snail, is expressed in this population (Jiang et al., 1998; Sefton et al., 1998). Indeed, Snail is capable of inducing a complete EMT in mammalian epithelial cells (Cano et al., 2000), and it should be noted that Snail null mutant mice die at gastrulation due to defects in EMT at the stage when the early mesoderm delaminates from the primitive streak (Carver et al., 2001). Thus, in neural crest development, it seems that the role played by Slug in the chick, may be performed by Snail in the mouse. However, owing to the early lethality of the mutants, the direct role of Snail in neural crest specification remains to be tested in the mouse.

We have previously shown that, in Xenopus, Snail is one of the earliest genes expressed in the prospective neural crest (Essex et al., 1993; Mayor et al., 1993). However, no direct comparison between the expression of Snail and Slug has been performed. In addition, Snail function has not been fully analysed in this population. In this study we have generated functional derivatives of Xenopus Snail in order to study its role in neural crest development. Our results show that Snail acts as a transcriptional repressor, whose activity is required for the early specification and migration of the neural crest. Interestingly, the activation of Snail is sufficient to trigger the expression of all the neural crest markers tested, including Slug. Expression of these markers could be induced both in whole embryos and in animal caps in the absence of neural and mesodermal markers. We propose that Snail lies upstream of Slug in the genetic cascade responsible for neural crest specification.

\section{MATERIALS AND METHODS}

\section{Embryonic manipulation}

Embryos were obtained from adult Xenopus laevis by standard hormone-induced egg laying and artificial fertilisation (Villanueva et al., 2002). Embryos were staged according to Nieuwkoop and Faber (Nieuwkoop and Faber, 1967) and dissections were carried out using eyebrow knives as indicated previously (Mancilla and Mayor, 1996).

\section{Plasmid constructs}

Inducible DNA constructs were prepared by fusing the entire coding regions of Xsnail (amino acid residues 1-259), Xslug (amino acid residues 1-266), chick Snail (amino acid residues 1-256) and Slug (amino acid residues 1-268) to the ligand binding domain of the human glucocorticoid receptor (GR, amino acid residues 512-777) (Fig. 1). Coding sequences were amplified by PCR using the following primers (see Fig. 1A-D):

Xsnail, 5'ATGCCCCGGTCATTTCTGGTC $3^{\prime}$ and 5'GAGCTCCGTGGGCCACCGTGCACC 3';
Xslug, 5'CCCAGTGAATTCATGCCACGATCTTTTCTG 3' and 5' TACTGGAGCTCCATGTGCTACACAGCA3';

chick Snail, 5'CCCAGTGAATTCATGCCGCGCTCGTTCC $3^{\prime}$ and 5' TACTGGAGCTCCGCGTGCCCCTGAGC 3'; and

chick Slug, 5'CCCAGTGAATTCATGCCACGCTCCTTCCTG 3'.

GR was obtained by PCR from pSP64T-MyoD-GR (Kolm and Sive, 1995) using the primers 5'-GGCGCCGAGCTCCCCTCTGAAAATCCTGG-3' and 5'GCGGGCTCGAGCCACTTTTGATGAAACAGAAC-3'.

The PCR products were purified and cloned into pGEM-T Easy vector (Promega). EcoRI/SacI sites in the Snail and Slug genes and, $\mathrm{SacI} / \mathrm{XhoI}$ sites from GR were used to fuse the two, and ligate them into an EcoRI/XhoI-digested pCS2+ vector (donated by Dr D. Turner). The Xsnail (amino acid residues 134-259) and Xslug (amino acid residues 72-266) zinc-finger coding regions were amplified using the primers 5'-GAATTCCAAGCACAAACAGTTGCACTG-3' and $5^{\prime}$ GAGCTCCGTGGGCCACCGTGCACC-3', and 5'-CCCAGTGAATTCATGCCACGATCTTTTCTG-3' and 5'-TACTGGAGCTCCATGTGCTACACAGCA-3' respectively, and cloned into pGEM-T Easy vector. The EcoRI and $S a c I$ sites (underlined) were used to fuse them to GR (Fig. 1E,F). To generate the XsnailN or XslugN constructs (Fig. $1 \mathrm{G}, \mathrm{H}$ ), the N-terminal part of Xsnail (amino acid residues 1-145) and Xslug (amino acid residues 1-151) were amplified by PCR using the primers 5'-GAATTCCATGCCCCGGTCATTTCTGG-3' and 5'GAGCTCTGGGAGTCACAGTGCAACTG-3', and 5'-GAATTCAATGACCCGATCTTTTCTGG-3' and 5'-GAGCTCTGGGCGTCGCAATGCAGCTG-3',respectively. The PCR products were purified and cloned into pGEM-T Easy vector, EcoRI/SacI-digested and ligated with a SacI/XhoI-digested GR fragment into pCS2+ vector digested with EcoRI/XhoI.

To generate the transcriptional activator and repressor chimeras, the Xsnail and Xslug zinc-finger DNA-binding domains obtained as described above were fused to the engrailed repressor domain (EnR) or to the E1A transactivator domain in the $\mathrm{pCS} 2+\mathrm{EnR}$ and $\mathrm{pCS} 2+\mathrm{E} 1 \mathrm{~A}$ plasmids (donated by N. Papalopulu).

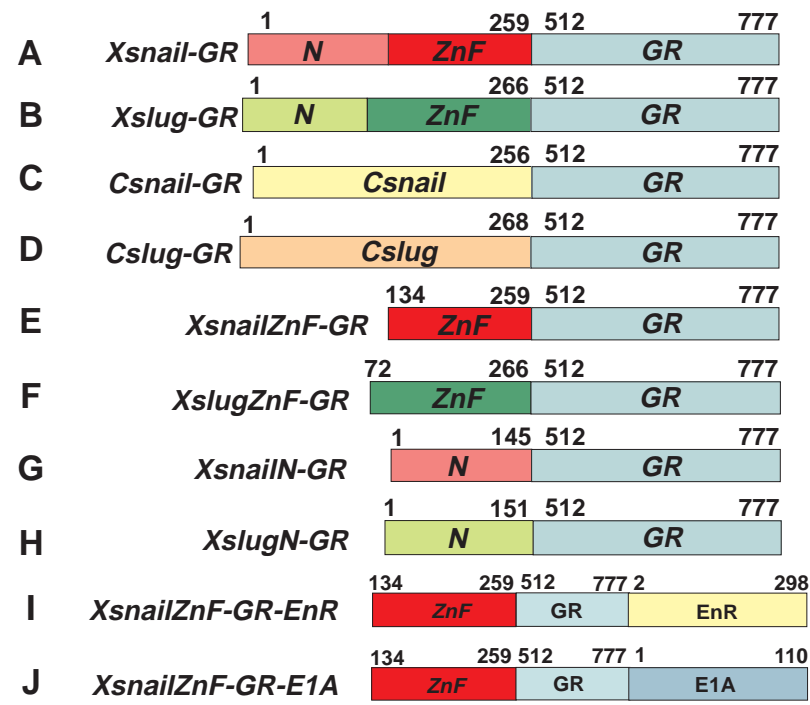

Fig. 1. Snail fusion proteins. The constructs used to produce the Snail fusion proteins are represented in this figure. The numbers above the bars indicate the amino acid number in the wild-type protein. Red is used for Xenopus Snail, green for Xenopus Slug, yellow for chick Snail and orange for chick Slug. N, amino terminal; $\mathrm{ZnF}$, zinc-finger region; GR (light blue), glucocorticoid receptor domain; EnR (yellow), transcriptional repressor domain of the Engrailed protein of Drosophila; E1A (dark blue), transcriptional activator domain. See Materials and Methods for details. 
To obtain the E1A fusion protein (Fig. 1J), the pCS2+E1A vector and Xsnail zinc-finger fragment fused to GR were digested with EcoRI/XhoI and ligated. The EnR fusion construct (Fig. 1I) was generated by exchanging the E1A domain, excised with XhoI and $K p n I$, from the pCS2+ZnFXsnailGR-E1A or pCS2+ZnFXslugGRE1A with the EnR-coding sequence, excised with the same enzymes, from the pCS2+EnR vector. All fusion constructs were sequenced on both strands at junction sites by automated DNA sequencing (BRC, Cornell University, Ithaca, NY). All cDNAs were linearized and transcribed with a GTP cap analog (New England Biolabs) using SP6, T3 or T7 RNA polymerases, as described elsewhere (Harland and Weintraub, 1985). After DNAse treatment, RNA was phenolchloroform extracted, ethanol precipitated and resuspended in DEPCtreated distilled water.

\section{RNA microinjection, lineage tracing and dexamethasone induction}

Dejellied embryos were placed in 75\% NAM containing 5\% Ficoll and one blastomere of two-cell stage embryos was injected with differing amounts of capped mRNA containing 1-3 $\mu \mathrm{g} / \mu \mathrm{l}$ lysine fixable fluorescein dextran (40,000 $M_{\mathrm{r}}$; FDX, Molecular Probes) as a lineage tracer. For animal cap assays, mRNA was injected into the animal side of the two bastomeres of two-cell stage embryos. Approximately 8-12 nl of diluted RNA was injected into each embryo. Ethanol-dissolved dexamethasone $(10 \mu \mathrm{M})$ was added to the culture medium at stages 12.5 or 16 and maintained until the embryos were fixed. To control the possible leakage of inducible chimeras, a sibling batch of embryos were cultured without dexamethasone and processed for in situ hybridisation.

\section{In situ hybridisation and immunohistochemistry}

Antisense probes containing Digoxigenin-11-UTP (Roche Biochemicals) were prepared for FoxD3 (Sasai et al., 2001), Xtwist (Hopwood et al., 1989), Sox2 (Dr RM Grainger, personal communication) and cytokeratin $X k 81 A$ (Jonas et al., 1985) by in vitro transcription. In order to avoid cross hybridisation between Snail and Slug, and to distinguish between endogenous expression and exogenous mRNA, the probes for Xsnail (Essex et al., 1993) and Xslug (Mayor et al., 1995) were synthesised from 3' untranslated regions prepared with the following primers: 5'-GCACAATGGACTCCTTAAATTCCTG-3' (upstream) and 5'- GTGACCGGGTGCTCATTGTG-3' (downstream), and 5'-GTTTACCAGGACTTAACACCTCC-3' (upstream) and 5'-GCATTCCCTTAAACCCTTCTTGG-3' (downstream), respectively.

Specimens were prepared, hybridised and stained according to Harland (Harland, 1991) with modifications (Mancilla and Mayor, 1996). Detection of labelled antisense probes was performed using alkaline-phosphatase conjugated anti-digoxigenin Fab fragments (Roche Biochemicals) and with NBT/BCIP (purple) as substrate. We have designed a new protocol to detect the lineage tracer in combination with the in situ hybridisation procedure. First, the in situ hybridisation alkaline-phosphatase reaction was stopped by incubation in methanol at $65^{\circ} \mathrm{C}$ for 1 hour, then embryos were rehydrated and blocked with $2 \%$ Roche blocking-reagent before incubating with alkaline phosphatase conjugated anti-Fluorescein Fab fragments (Roche Biochemicals). The phosphatase activity resulting from the lineage tracing was detected using BCIP (green) as substrate. Rabbit Polyclonal anti-phosphohistone-3 from Upstate Biotechnology was used to analyse mitotic cells according the method described elsewhere (Turner and Weintraub, 1994).

\section{RNA isolation and RT-PCR analysis}

Total RNA was isolated from embryonic tissue by the guanidine thiocyanate/phenol/chloroform method (Chomczynski and Sacchi, 1987), and cDNAs were synthesised using AMV reverse transcriptase (Roche Biochemicals) and oligo(dT) primer. The primers designed for this study were: Xslug, 5'- GTTTACCAGGACTTATCACCTCC-3' (upstream) and 5'-GCATTCCCTTAAACCCTTCTTGG-3' (downstream); Xsnail, 5'-GCACAATGGACTCCTTAAATTCCTG-3' (upstream) and 5'-GTGACCGGGTGCTCATTGTG-3' (downstream).

The Ets 1 primer sequences used were those previously (Meyer et al., 1997). Xtwist, Ncam, Sox2, Xbra, H4 and BMP4 primer sequences were obtained from the website of $\mathrm{Dr}$ Eddy De Robertis (http://www.hhmi.ucla.edu/derobertis/protocol_page/oligos.PDF).

Zic5 primer sequences were as described previously (Nakata et al., 2000). PCR amplification with these primers was performed over 28 cycles and the PCR products were analysed on $1.5 \%$ agarose gels. As a control, PCR was performed with RNA that had not been reversetranscribed to check for DNA contamination.

\section{RESULTS}

\section{Snail expression at stages of neural crest induction}

The expression pattern of Snail and Slug in Xenopus embryos have been described previously (Essex et al., 1993; Mayor et al., 1993; Mayor et al., 1995; Linker et al., 2000). We show a side-by-side comparison at the stages of neural crest induction. The expression of Snail commences in the dorsal marginal zone just before gastrulation (stage 9; Fig. 2A). It is at this midgastrula stage (between stage 11 and 11.5) when the onset of Snail expression can be detected in the ectoderm, in an arc that surrounds the prospective neural plate (Fig. 2B,D). The comparison with Slug expression shows that whereas Snail could be detected at the neural plate border from stage 11 (Fig. 2B,D), Slug expression is only detectable from stage 12.5 (Fig. $2 \mathrm{C}, \mathrm{F})$. From stage 12, the anterior domain of Snail expression is downregulated, while its levels are increased in the prospective neural crest region, where Slug transcripts can be detected (Fig. 2E-G).

In Xenopus, the ectoderm is formed of two layers: the superficial or sensorial layer, and the deep layer where the neural crest is generated. At the mid neurula stage (Fig. 2G), the cells expressing Snail in the outer band correspond to the crest cells, while the cells in the thin inner band (white arrowhead in Fig. 2G) will end up in the roof plate of the neural tube. In summary, Snail is the earliest known gene to be expressed in the prospective neural crest, preceding Slug expression. Snail early expression is restricted to the neural plate border, including the prospective neural crest region and the anterior neural folds, which are fated to become the forebrain (Fig. 2H,I).

\section{Snail promotes neural crest specification}

In order to analyse the influence that Snail might have on neural crest development, we have used an inducible Snail construct. The use of such an inducible construct is important as Snail is expressed in the mesoderm at early stages, and any effects on this tissue could indirectly influence the neural crest. Thus, one blastomere of two-cell stage embryos was injected with 50 to $700 \mathrm{pg}$ of XsnailGR mRNA, and the expression of several neural crest, neural plate and epidermal markers was analysed at the neurula stage. A reproducible phenotype was observed when $>100 \mathrm{pg}$ of mRNA were injected. This phenotype involved the expansion of the area in which neural crest markers were expressed [Snail, 85\% of the embryos showed expansion ( $n=107)$; Slug, 76\% ( $n=96)$; Zic5, 63\% ( $n=97$, not shown); Twist, 67\% (n=86) and FoxD3, 62\%, $(n=85)]$, and a reduction in the area over which the neural plate 

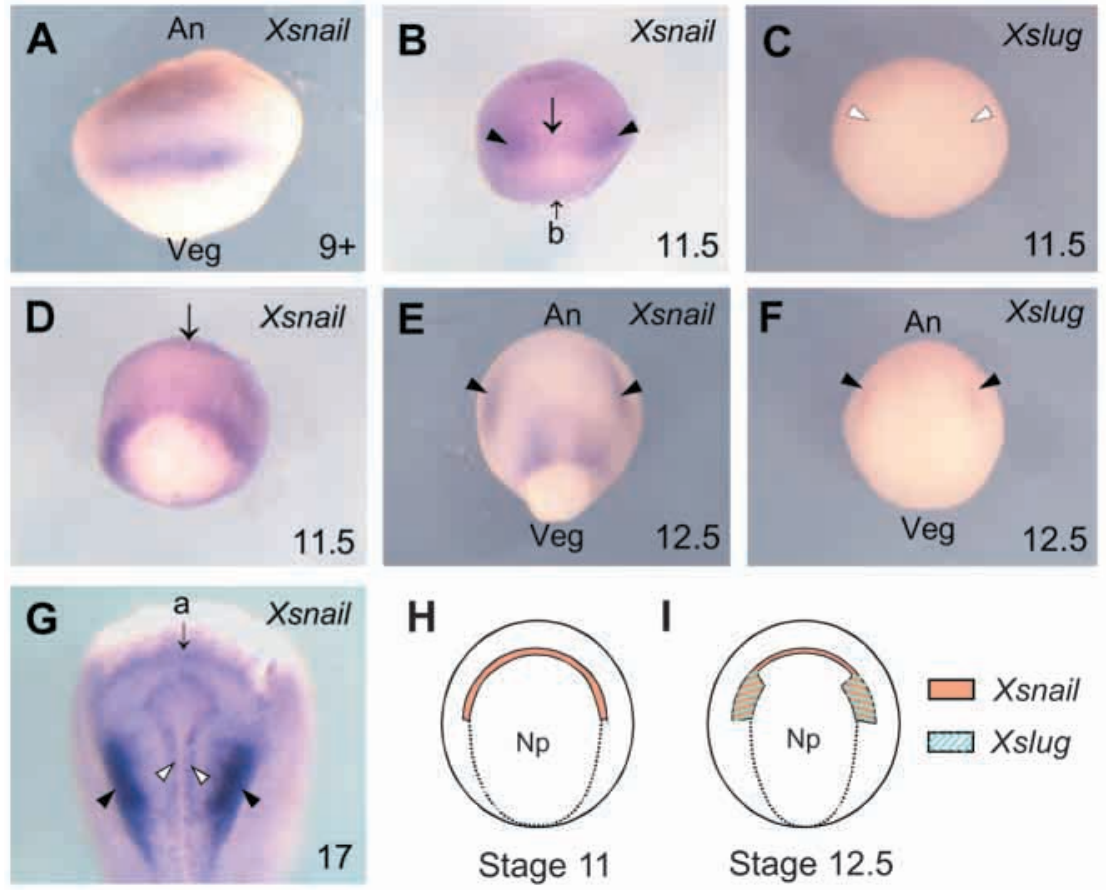

Fig. 2. Expression pattern of Snail and Slug analysed by whole-mount in situ hybridisation. (A) Dorsal view of a stage 9 embryo. An, animal pole; Veg, vegetal pole. Notice the expression in the dorsal marginal zone. (B) Dorsal view of a stage 11.5 embryo. b, dorsal blastopore lip. Snail is expressed in the prospective neural crest (arrowheads) and in a continuous band at the anterior border of the neural plate (arrow). (C) Same embryo as in B (stage 11.5) but hybridised for Slug expression. No expression is seen in the ectoderm (white arrowheads). (D) Dorsovegetal view of the embryo shown in B. Arrow indicates Snail staining in the anterior ectoderm. This is also visible around the blastopore lip in the marginal zone, apart from the dorsal region where Snail has been switched off. (E) Dorsal view of a stage 12.5 embryo. Expression is visible in the mesoderm near the blastopore and in the prospective neural crest (arrowhead). An, animal pole; Veg, vegetal pole. (F) Earliest Slug expression. Dorsal view of a stage 12.5 embryo. Expression in the prospective neural crest (arrowheads). An, animal pole; Veg, vegetal pole. (G) Anterior view of a stage 17 embryo. Expression in the superficial (white arrowheads) and in the deep (black arrowheads) layers of the ectoderm and in the anterior neural fold (a, arrow). (H,I) Schematic representation of Snail and Slug expression at stages 11 and 12.5. Anterior is upwards and posterior is downwards.

marker Sox2 $(87 \%, n=92)$ and the epidermal marker cytokeratin (89\%, $n=65)$ were expressed (Fig. 3). It is interesting to note that the expression of cytokeratin almost completely disappeared from the injected side of the embryo. This observation suggests that the expansion of the neural crest domain results from a transformation of the epidermis and some of the neural plate region into prospective neural crest. This is similar to the mechanism that has been proposed to explain the increase in neural crest markers observed after Slug overexpression (LaBonne and Bronner-Fraser, 2000; Mayor et al., 2000). However, it is also possible that as a result of the injection, an increase in the rate of neural crest cell proliferation was triggered. In order to rule out this possibility, we repeated the Snail and Slug injections in the presence of the inhibitors of cell proliferation hydroxyurea and aphidicolin (HUA) (Harris and Hartenstein, 1991; Sharpe and Goldstone, 1997). This inhibition did not affect the expression or the expansion of the territory in which the neural crest markers were observed after injecting the SnailGR or SlugGR construct: Snail $(92 \%, n=42 ; 82 \%$, $n=45)$ or Slug $(85 \%, n=49 ; 85 \%, n=42$ ) (Fig. 4). Cell proliferation can be assessed by the detection of a phosphorylated form of $\mathrm{H} 3$ histone (Fig. 4F). In the presence of HUA, its signal is barely detectable (Fig. 4E). Thus, we conclude that the expansion in the neural crest territory is not due to an increase in cell proliferation but instead is the consequence of generating additional neural crest cells.

To address if Snail expression was sufficient to induce neural crest markers we performed animal caps experiments. Embryos were injected at the one-cell stage with $1 \mathrm{ng}$ of Snail mRNA and at the blastula stage (stage 9), the animal caps were dissected out and cultured until they reached the equivalent of stage 20, when the expression of different markers was analysed by RT-PCR. In these experiments, the expression of $\mathrm{H} 4$ histone was used as a loading control. In whole embryos, the normal expression of all the markers tested was observed, while in uninjected control animal caps the expression of specific markers could not be detected (Fig. 5A). The injection of $1 \mathrm{ng}$ of Snail mRNA was sufficient to trigger the expression of the neural crest markers Snail, Slug, Twist, Zic5 and Ets 1, but not that of neural plate markers, such as NCAM or Sox2, or mesodermal markers, such as Xbra (Fig. 5A). This result suggests that Snail itself is capable of converting ectodermal cells into neural crest cells, and that it could lie upstream of the genetic cascade required for neural crest specification.

Because our results show that Snail produces a strong reduction of epidermal markers, and it is known that the development of epidermis requires high levels of BMP activity, we tested whether Snail overexpression had an effect on BMP4 transcription. The expression of BMP4 was analysed in animal caps as described above and a clear inhibition of BMP4 expression was observed when the animal caps were injected with Snail (Fig. 5B).

\section{Inhibition of Snail activity blocks early neural crest specification}

In order to test whether Snail activity is required for neural crest development, we tested the effect of a dominant-negative construct in which the zinc-finger domain of Snail was fused to the GR element (XsnailZnFGR). This construct was designed to bind to the appropriate sequences in the promoter of target genes, but with the idea that it will be incapable of regulating transcription, as has been shown for this type of construct using the Slug gene (LaBonne and Bronner-Fraser, 2000; Mayor et al., 2000). Its injection blocked the expression of Snail (82\% of the embryos, $n=57)$, Slug $(77 \%, n=67)$, FoxD3 (83\%, n=55), Twist (83\%, n=53) and Zic5 (77\%, n=43, not shown) (Fig. 6A-D). In order to show the specificity of this dominant negative, we performed the following rescue 


\section{Xsnail-GR}

\section{$N$}
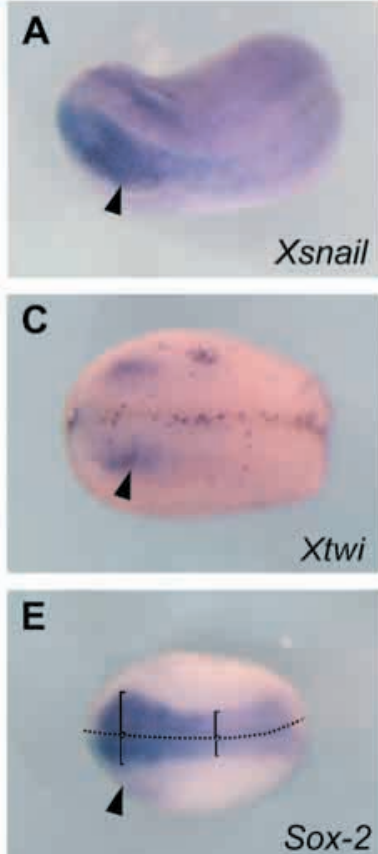

Sox-2
GR
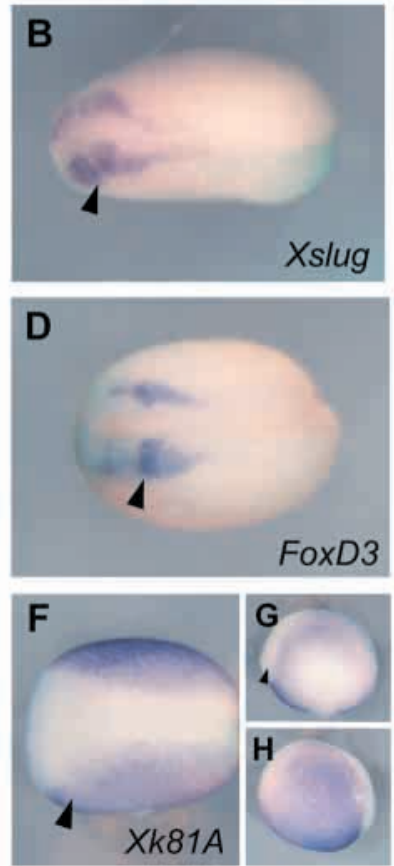

Fig. 3. Snail participates in the early specification of the neural crest. One blastomere of a two-cell stage embryo was injected with $700 \mathrm{pg}$ of Xsnail-GR mRNA, treated with dexamethasone at stage 12.5, fixed at stage 19, and the expression of several genes analysed. The arrowheads indicate the injected side that contained FLDx (see Materials and Methods). Anterior is towards the left. (A-D) Neural crest markers. Notice the expansion of the markers on the injected side. (A) Snail expression. (B) Slug expression. (C) Twist expression. (D) FoxD3 expression. (E) Expression of the neural plate marker Sox 2 , is reduced on the injected side. The broken line indicates the dorsal midline and the brackets indicate the width of the neural plate. (F) Expression of the epidermal marker Cytokeratin 81A (dorsal view), is almost completely inhibited on the injected side. $(\mathrm{G}, \mathrm{H})$ Lateral views of the same embryo where the inhibition of Cytokeratin expression is better assessed. G corresponds to the injected side.

experiment. Embryos were co-injected with the same amount of mRNA that codes for XSnailZnfGR (dominant negative) and XsnailGR (wild type) and the expression of several neural crest markers was analysed. A strong rescue in the expression of the neural crest markers was observed (Fig. 6E,F). The injected embryos show a normal expression of Slug $(83 \%$ of normal expression in the injected side, $n=54)$, Snail $(96 \%$, $n=27)$, Zic5 (91\%, n=33, not shown) and FoxD3 (84\%, $n=31)$. Thus, by inhibiting Snail function the early specification of the neural crest was blocked as determined by the analysis of five different markers.

Owing to the sequence similarity between Snail and Slug in the finger region (Manzanares et al., 2001), the possibility exists that the injection of XsnailZnFGR could affect the function of both genes. Thus, we decided to generate another dominant-negative construct by fusing the highly divergent Nterminal domain of Snail to the GR element (XsnailNGR). This construct will not be able to bind DNA but should be capable of binding other proteins required for the transcriptional activity of these factors, as shown for a similar FoxD3 construct (Sasai et al., 2001). We considered that XsnailNGR would serve as a more specific construct to test the effects of expressing dominant-negative Snail constructs. Indeed, injection of the N-terminal dominant-negative version also blocked the expression of Slug $(89 \%, n=47)$ and FoxD3 $(83 \%$, $n=46$ ) (Fig. 6G,H). Nevertheless, and in order to check unambiguously the specificity of this construct, we carried out a rescue experiment similar to that described for XsnailZnFGR. The co-injection of the XsnailNGR (dominant negative) together with XsnailGR (wild type) produces a rescue in the expression of the neural crest markers analysed (61\% of normal Slug expression, $n=26$; and $56 \%$ of normal FoxD3, n=25) (Fig. 6I,J). Thus, taken together our results show that Snail activity can be specifically blocked by two types of dominant negatives and that Snail is required to control the expression of all the neural crest markers tested.

\section{Snail lies upstream of Slug in the genetic cascade leading to the neural crest development}

The temporal appearance of Snail and Slug in the neural crest together with the ability of Snail to upregulate Slug expression in whole embryos and in animal caps suggest that Snail could

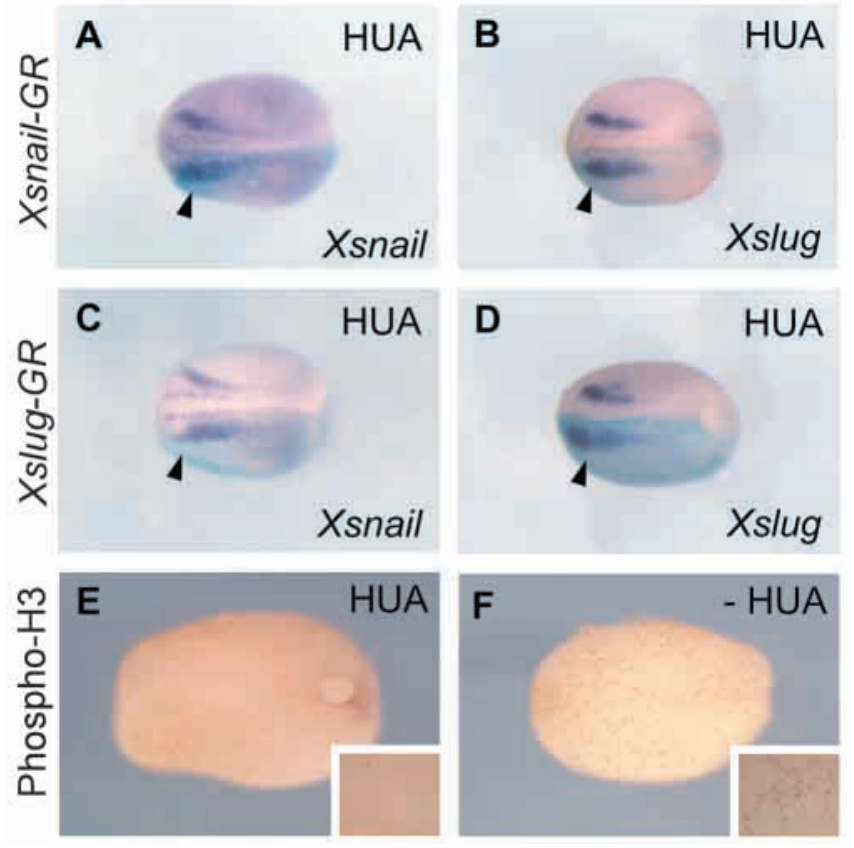

Fig. 4. The expansion of the neural crest territory induced by Snail or Slug does not require cell proliferation. One blastomere of a two-cell stage embryo was injected with 700 pg of Xsnail-GR mRNA (A,B) or Xslug-GR (C,D), treated with dexamethasone and HUA at stage 12.5, fixed at stage 19 and the expression of the neural crest markers Snail (A,C) and Slug (B,D) analysed. The injected side, which can be recognised by the blue FLDx staining, is indicated by the arrowhead. Note the expansion in the expression of the neural crest markers on the injected side. (E) HUA treated and (F) control embryos stained for histone $\mathrm{H} 3$ to verify the blockade in cell proliferation induced by the treatment. The inset shows a higher magnification of the embryos. Note the staining in absence of HUA treatment, but the lack of staining after HUA treatment. 


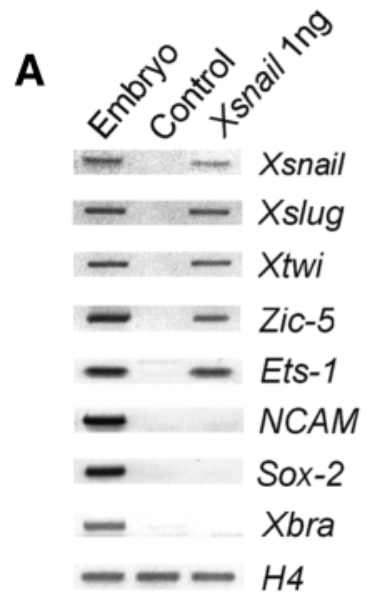

Fig. 5. Snail is able to activate the expression of neural crest markers and block BMP4 expression. Embryos were injected at the one-cell stage with 1 ng of Snail mRNA. At stage 8, the animal caps were dissected and cultured until the equivalent of stage 20, when mRNA was isolated and assayed by RT-PCR as described in the Materials and Methods. Embryo: whole embryo showing the expression of all the markers. H4 was used as a loading control. Control: uninjected animal caps. Xsnail $1 \mathrm{ng}$ : animal caps taken from embryos injected with Snail mRNA. (A) Note that Snail is able to induce early and late neural crest markers in the absence of neural plate and mesodermal markers. (B) Note the inhibition in the expression of BMP4 after Snail mRNA injection.

be upstream of Slug in the genetic cascade that specifies the neural crest cells. If this is the case, the effect of a dominantnegative Snail could be rescued by Slug, but that of a dominantnegative Slug should not be rescued by Snail. To test this prediction, embryos were co-injected with XsnailZnFGR (dominant negative) and SlugGR (wild type) and the expression of different neural crest markers was analysed. An almost complete rescue of the neural crest expression was observed (Fig. 7A,B). Injected embryos showed a normal expression of Slug (94\%; $n=69)$, Snail $(85 \% ; n=41)$, Zic5 (91\%; $n=46)$ and FoxD3 (88\%; $n=42)$. A similar rescue was observed when the XsnailNGR dominant negative was coinjected with SlugGR (not shown). This suggests that Slug activity is downstream of Snail. To further confirm this finding, we developed a new dominant-negative Slug construct similar to XsnailNGR. The highly divergent N-terminal domain of Slug was fused to the GR element (XslugNGR) and this construct was injected into one blastomere of a two-cell stage embryo. This injection blocked the expression of Slug itself (86\% of the embryos, $n=43)$ and that of FoxD3 $(74 \%, n=46)$ (Fig. 7C,D) in a similar manner to that previously described for the dominant-negative zinc-finger Slug construct (LaBonne and Bronner-Fraser, 2000; Mayor et al., 2000). However, this inhibition in the expression of the neural crest markers was not efficiently rescued by co-injection of SnailGR. Only 30\% of the injected embryos showed a normal expression of Slug $(n=23$; Fig. 7E) and $38 \%$ of them showed normal FoxD3 expression ( $n=38$; Fig. 7F). By contrast, the rescue of the Slug dominant-negative by SlugGR was apparent in $77 \%$ and $72 \%$ of the embryos with respect to Slug $(n=44)$ and FoxD3 expression $(n=43)$, respectively. Thus, the rescue of the Snail
XsnailZnF-GR
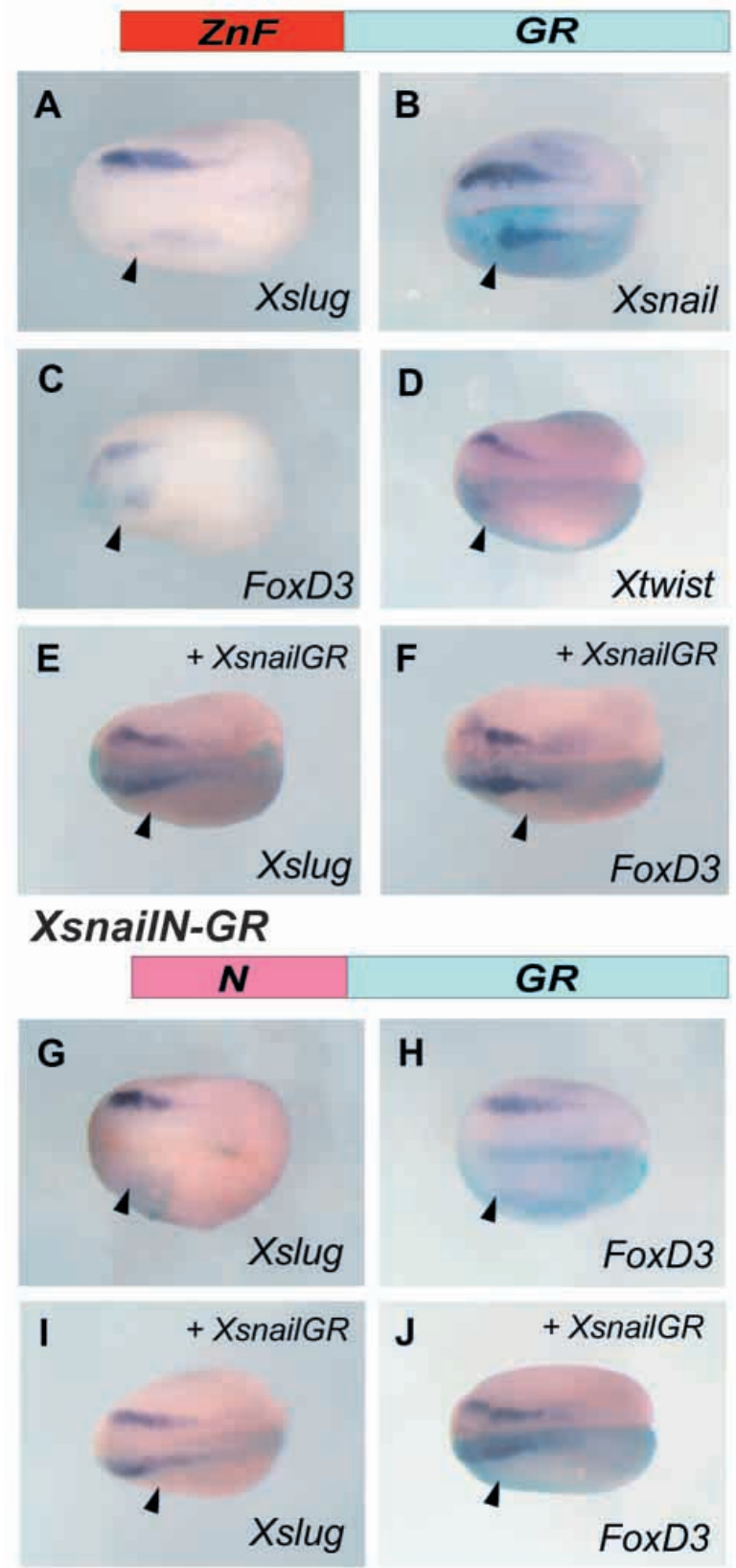

Fig. 6. Inhibition of Snail activity blocks the expression of neural crest markers. One blastomere of a 2-cell stage embryo was injected with $700 \mathrm{pg}$ of the different dominant negative constructs, treated with dexamethasone at stage 12.5 , fixed at stage 19 , and the expression of the neural crest markers analysed. The injected side is indicated by an arrowhead. (A-D) XsnailZnFGR: dominant-negative of the Snail zinc fingers. Note that the dominant negative construct inhibited the expression of all the neural crest markers analysed. (E,F) Rescue of XsnailZnFGR by XsnailGR: both mRNAs were injected in equivalent amounts and analysed as previously described. Note the normal expression of the neural crest markers in the injected side. $(\mathrm{G}, \mathrm{H})$ SnailN-GR: dominant-negative using the Snail $\mathrm{N}$-terminal domain. Note that the dominant-negative constructs inhibited the expression of all the neural crest markers analysed. (I,J) Rescue of XsnailNGR by XsnailGR: both mRNAs were injected in equivalent amounts and analysed as previously described. Note the normal expression of the neural crest markers in the injected side. 


\section{XsnailZnF-GR}

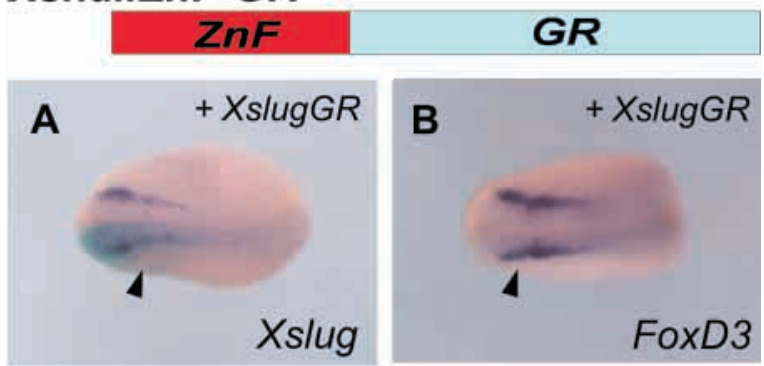

XslugN-GR
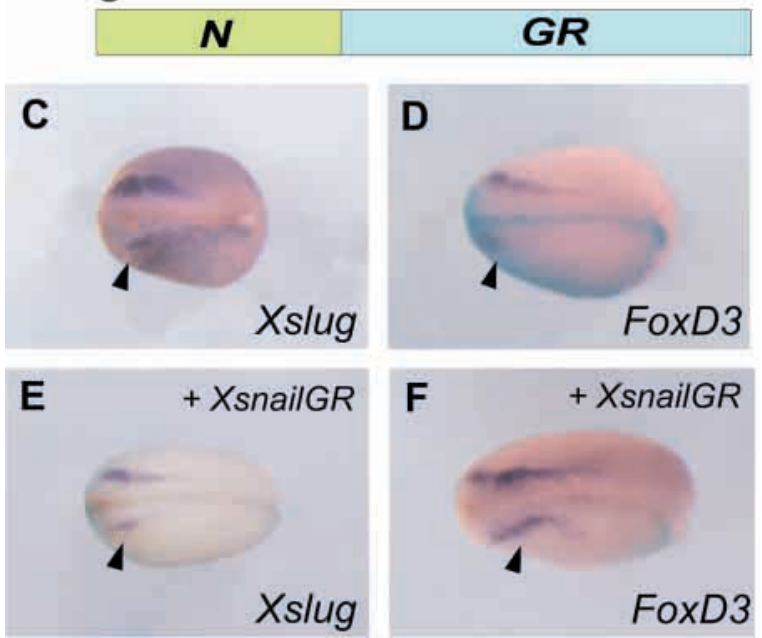

Fig. 7. Snail lies upstream of Slug in the cascade leading to neural crest development. One blastomere of a two-cell stage embryo was co-injected with the different dominant-negative constructs and the wild type, treated with dexamethasone at stage 12.5 , fixed at stage 19 and the expression of the neural crest markers analysed. The injected side is indicated by an arrowhead. (A,B) XsnailZnFGR rescued by XslugGR: the effect of the zinc fingers dominant-negative Snail construct was rescued by co-expression of Slug. Note the normal expression of neural crest markers in the injected side. (C,D) Effect of injecting XslugNGR dominant-negative construct. Note the inhibition in the expression of the markers in the injected side. (E,F) Co-injection of XslugNGR and XsnailGR: note that the effect of the dominant-negative Slug construct can not be rescued by coexpression of Snail.

dominant-negative by Slug, and the difficulty to rescue Slug activity by Snail indicates that Snail is upstream of Slug in the genetic cascade that specifies the neural crest in the ectoderm.

\section{Snail functions as a transcriptional repressor}

In order to better understand the molecular mechanisms that underlie the activity of Snail during neural crest development, the zinc-finger region of Snail was fused to the activation domain of E1A or the repressor domain from Drosophila engrailed. Both these constructs were fused to the GR element to make them inducible and they were called XsnailZnFGRE1A and XsnailZnFGrEnR, respectively. One blastomere of a two-cell stage embryo was injected with one or other of these constructs and the expression of several neural crest markers analysed at the neurula stage. The injection of the repressor construct resulted in an enlargement of the territory expressing Slug (81\% of expansion, $n=44)$, Snail (88\%, $n=44)$, FoxD3 (85\%, n=41), Zic5 (78\%, $n=46$, not shown) and Twist (78\%, n=46) (Fig. 8A-D). Conversely, injecting the activator construct led to an inhibition of the expression of Slug (86\% of inhibition, $n=45)$, Snail $(82 \%$, $n=58)$, FoxD3 (81\%, n=48), Zic5 (80\%, n=56, not shown) and Twist $(76 \%, n=50)$ (Fig. 8E-H). Thus, as the repressor construct produced the same phenotype as wild-type Snail, and the opposite effect was produced by the activator construct, we concluded that Snail probably functions as a transcriptional repressor in this system.

\section{Snail has a role on neural crest migration}

As Snail is also expressed during the migration of neural crest cells, we analysed whether it might also influence this process.
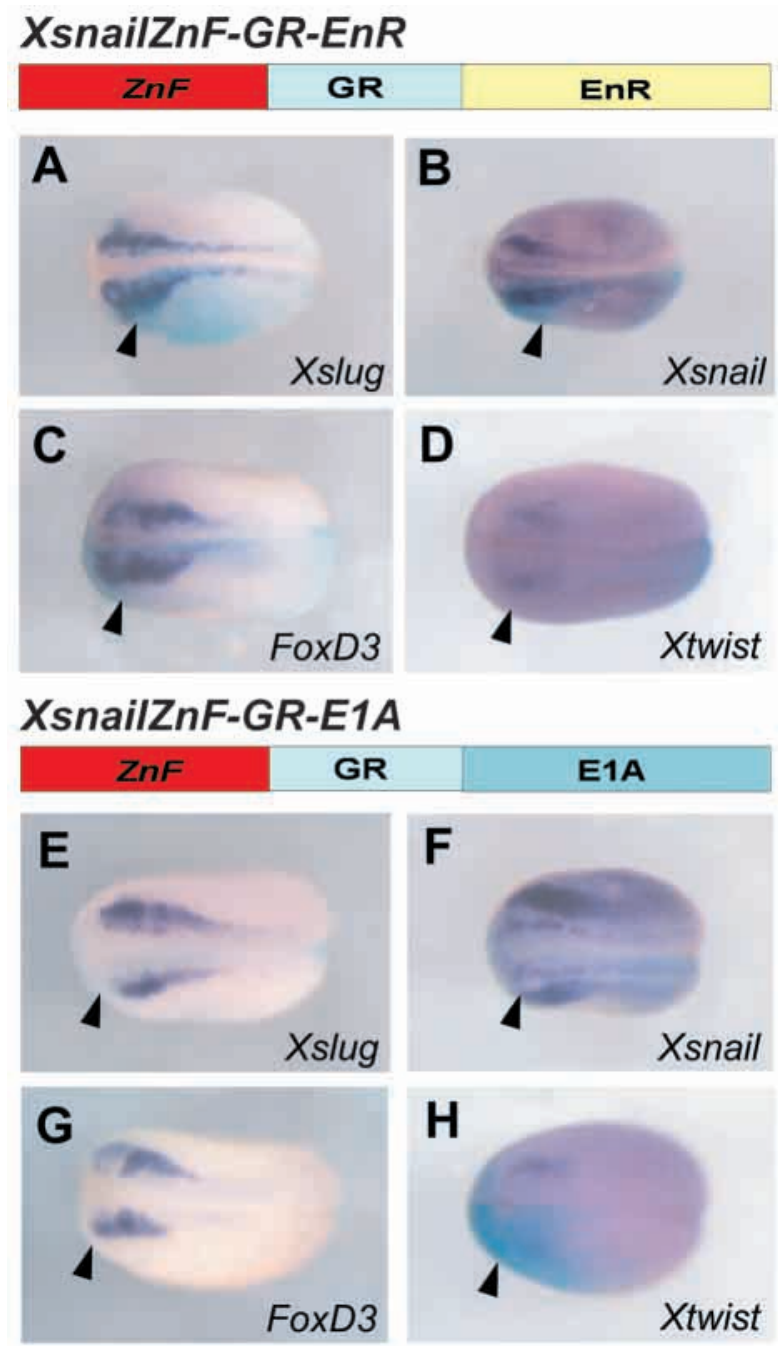

Fig. 8. Snail functions as a transcriptional repressor. One blastomere of a two-cell stage embryo was injected with $700 \mathrm{pg}$ of a Snail repressor construct (A-D) or the Snail activator construct (E-H), treated with dexamethasone at stage 12.5 , fixed at stage 19 , and the expression of neural crest markers analysed. Arrowhead, injected side. Note that the Snail repressor construct (XsnailZnF-GR-EnR) produced an expansion of the neural crest markers on the injected side (A-D), while the Snail activator lead to an inhibition in the expression of the markers (E-H). 


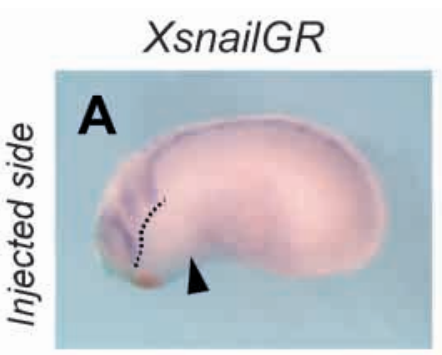

ZnFXsnailGR
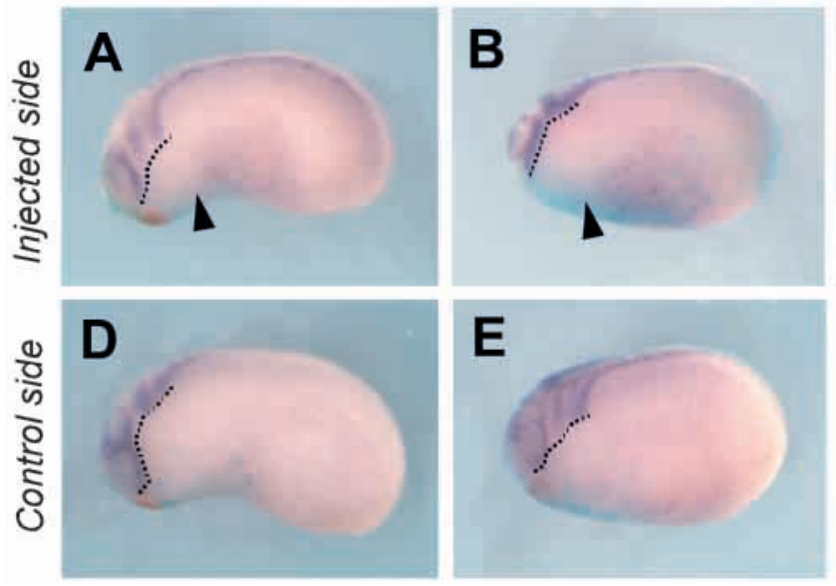

\section{XslugGR}
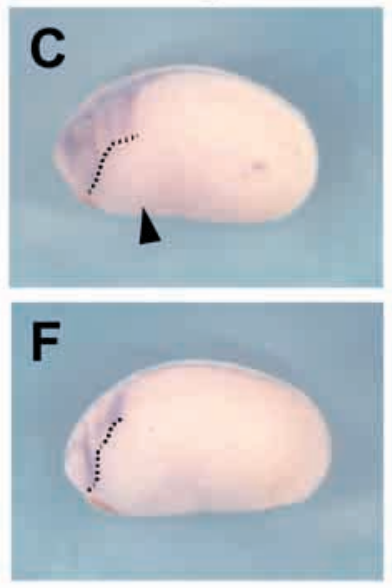

Fig. 9. Snail controls neural crest migration. One blastomere of a two-cell stage embryo was injected with $700 \mathrm{pg}$ of SnailGR (A,D), its dominant negative $(\mathrm{B}, \mathrm{E})$ or SlugGR $(\mathrm{C}, \mathrm{F})$ treated with dexamethasone at stage 16, fixed between stages 22 and 23, and the expression of the neural crest markers Slug analysed. (A-C) Injected side (arrowhead). (D-F) Uninjected side of the embryos shown in A-C. The leading edge of migration is indicated with a broken line. Note that SnailGR (A,D) and SlugGR $(\mathrm{C}, \mathrm{F})$ produces a stronger migration in the injected side; while the injection of ZnFXsnailGR (B,E) leads to an inhibition in the migration of the crest cells.
Embryos injected with XsnailGR and with XsnailZfGR (dominant negative) were allowed to develop to the midneurula stage (stage 16-17) when the neural crest is specified and expresses both Slug and Snail (Mayor et al., 1995; Mancilla and Mayor, 1996). When the injected embryos were treated with dexamethasone at stage 16, and the expression of neural crest markers was analysed at stage $22-23$, a prominent effect on neural crest migration was observed. The population of migrating crest expressing Slug (81\% of embryos with an increase in the injected side, $n=43$; Fig. 9A,D) and Snail (82\%, $n=51$, not shown) increased following injection of XsnailGR. In addition, injection of XsnailZfGR resulted in a reduction in the migration of the crest cells $(67 \%$ of embryos with a reduction in the migration in the injected side, $n=48$; Fig. 9B,E) or Snail $(74 \%, n=46$, not shown). These results are similar to those observed when the Slug dominant-negative construct was used previously in equivalent experiments (LaBonne and Bronner-Fraser, 2000). As Slug overexpression had not been examined previously at stages during which the neural crest is migrating, we also injected embryos with XslugGR and treated them in a similar way. Overexpression of Slug produced an increase in the migratory population of crest cells as seen by the expression of Slug (77\% of embryos with stronger migration in the injected side, $n=47$, Fig. 9C,F) and Snail (75\%, $n=51$, not shown). Thus, both Snail and Slug overexpression during the stages of crest migration gives rise to an increase in the migration of this population of cells.

\section{Ectopic expression of chick and mouse Snail and Slug in Xenopus embryos}

Slug has been shown to be important in triggering EMT during crest migration in the chick (Nieto et al., 1994; Del Barrio and Nieto, 2002). In the mouse, Snail rather than Slug is the gene expressed in this population, and Snail has also been shown to induce a complete EMT in mammalian epithelial cells (Cano et al., 2000). Thus, during neural crest development, it seems likely that in the mouse Snail might fulfil the role played by Slug in the chick. This suggests that these genes may be functionally equivalent and that both are capable of triggering EMT when expressed at the appropriate time and place (Del Barrio and Nieto, 2002). Because in Xenopus both genes are expressed in the premigratory neural crest, we wanted to know whether they are functionally equivalent in these cells and whether the chick genes have a similar effect when ectopically expressed in the frog. After injection of mRNA encoding the inducible forms of Slug and Snail from Xenopus and chick into one blastomere of a twocell stage Xenopus embryos, the expression of the neural crest marker Slug was analysed at the late neurula stage (stage 25). The expression of Slug was enhanced by the injection of 500 pg of mRNA for XsnailGR (83\% of enlargement of Slug in the injected side, $n=53)$, XslugGR $(88 \% n=55)$, chick SnailGR $(67 \%, n=45)$ and chick SlugGR $(82 \%, n=46)$ (Fig. 10). Thus, these injections produced an expansion of the neural crest territory and, an enhanced migration of the crest migration on

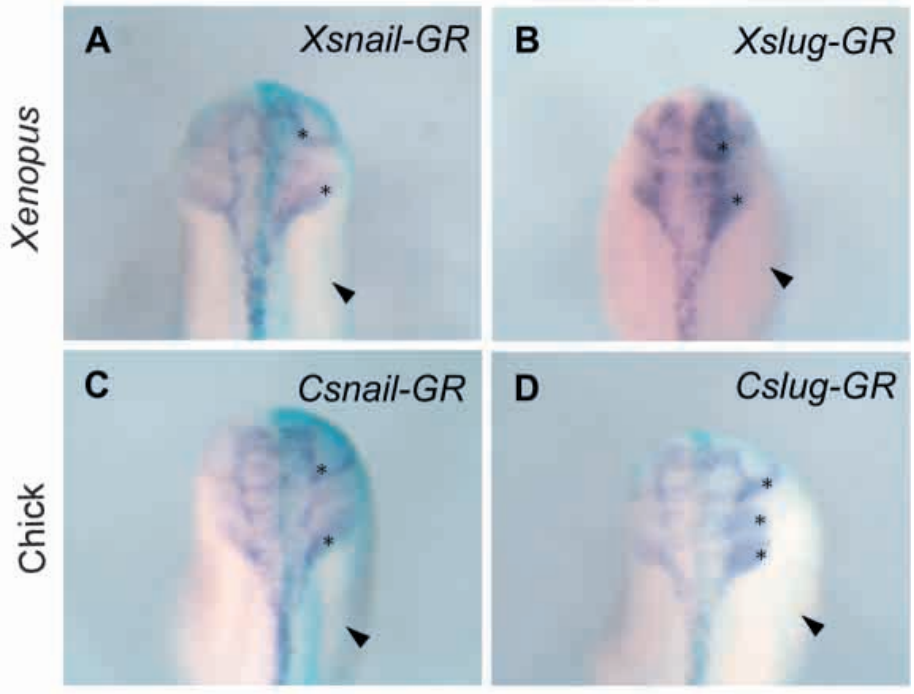

Fig. 10. Functional equivalence of the Snail genes assayed in Xenopus embryos. One blastomere of a two-cell stage embryo was injected with $500 \mathrm{pg}$ of mRNA encoding for different members of the Snail gene family, treated with dexamethasone at stage 12.5 , fixed at stage 25 , and the expression of the neural crest marker Slug analysed. Arrowhead indicates injected side. (A,B) Xenopus genes: Xsnail-GR (A) or XslugGR (B). (C,D) Chick genes: Snail-GR (C) or Slug-GR (D). Note that in all the injected sides of the embryos a more vigorous and larger population of migratory crest cells (asterisks). 
the injected side. Similar results (Fig. 10) were obtained after injection of mouse Slug $(65 \%, n=60)$ and Snail $(72 \%, n=51)$ (not shown). Thus, Snail and Slug from Xenopus, chick or mouse are functionally equivalent when overexpressed in Xenopus embryos.

\section{DISCUSSION}

\section{Snail family members during neural crest development}

Snail family members encode transcription factors of the zincfinger type (for a review, see Nieto, 2002). They are composed of a highly conserved $\mathrm{C}$ terminus, which contains from four to six zinc fingers, and a much more divergent $\mathrm{N}$ terminal region. The Snail family occupies a central role in mesoderm development from invertebrates to mammals, and plays a crucial role in neural crest formation in vertebrates (Nieto, 2002). Indeed, as a result of experiments where Slug expression was manipulated in Xenopus and chick, it has been proposed that this family member is a crucial gene for neural crest development (Nieto et al., 1994; LaBonne and BronnerFraser, 1998; Carl et al., 1999; Mayor et al., 2000; LaBonne and Bronner-Fraser, 2000; Del Barrio and Nieto, 2002). A lot of work has been focused on the involvement of Slug in neural crest development since it became the first gene to be implicated in the triggering of EMT during neural crest delamination (Nieto et al., 1994). Indeed, it is the only Snail family member expressed in the premigratory crest cells in the chick (Sefton et al., 1998) and we still believe that is involved in the specification of both head and trunk neural crest and in the migratory process in the head region (Del Barrio and Nieto, 2002). However, in Xenopus, both Slug and Snail are present in this population, where in fact Snail is expressed before Slug (Essex et al., 1993; Mayor et al., 1993; Linker et al., 2000) (this work). In the mouse, Snail is the family member that is expressed in neural crest precursors, however, mutant mice die too early to analyse its function in this tissue. These difficulties in studying this facet of Snail behaviour in the mouse has prompted us to examine the role it plays in neural crest development in Xenopus.

\section{Snail is the earliest marker for the neural crest}

Snail is the earliest marker of the neural crest described to date in Xenopus embryos, as it can be detected in the prospective neural crest from stage 11 onwards. All the other early neural crest markers such as Slug, FoxD3 and members of the Zic and Meis families are not detected in the neural folds until later on in development (Sasai et al., 2001; Nakata et al., 2000; Linker et al., 2000; Maeda et al., 2002). We show that, in addition, the initial expression of Snail in the ectoderm constitutes an arc that surrounds the neural/nonneural territory, including the anterior neural plate border. Just before the other early neural crest markers appear, or become confined to the crestproducing neural folds, Snail expression becomes weaker in the anterior region that will become part of the forebrain and does not form neural crest (Le Douarin and Kalcheim, 1999). This expression pattern is unique for Snail and perfectly compatible with the recently proposed inductionposteriorisation model for neural crest formation (Villanueva et al., 2002; Aybar and Mayor, 2002).

\section{Snail is required for the early specification of neural crest cells}

Having illustrated the intriguing expression pattern of Snail, we examined its role in neural crest development. Because Snail is also involved in mesoderm development, one must take care in interpreting the phenotypes generated by overexpressing this gene from the two-cell stage of development. A phenotype relevant to the neural crest could be the result of a previous effect on mesoderm development. For this reason, we have used inducible constructs that were activated around the time the neural crest is specified (Mayor et al., 1995; Mancilla and Mayor, 1996) and long after mesoderm specification (Smith et al., 1985).

Overexpression of Snail in whole embryos augmented the domain of expression of all the neural crest markers analysed at the expense of the adjacent ectoderm and neural plate. The amplification of this domain was not the result of an increase in cell proliferation as it was not inhibited when cell division was blocked. Thus, we infer that Snail has the ability to transform ectodermal cells into neural crest cells. This observation was further confirmed in animal cap assays, where expressing Snail mRNA alone was sufficient to trigger the expression of a series of early and late neural crest markers, in the absence of the expression of neural plate and mesodermal markers. This differs from results obtained when other early neural crest markers are expressed. The expression of genes such as Meis, Pbx, FoxD3 and Zic family members not only triggers the expression of neural crest markers, but also induces the expression of neural plate markers (Sasai et al., 2001; Nakata et al., 2000; Mizuseki et al., 1998; Nagai et al., 1997; Nakata et al., 1997; Nakata et al., 1998; Maeda et al., 2002). Furthermore, Slug overexpression was unable to trigger the expression of neural crest markers in animal cap assays as analysed by in situ hybridisation (LaBonne and BronnerFraser, 1998). Thus, it seems that Snail is the only gene that has been described to date that is capable of specifically transforming ectodermal cells into neural crest cells.

In support of Snail performing a central role in neural crest development, blocking Snail activity through the injection of dominant negative constructs produced a complete inhibition in the expression of neural crest markers. One of these constructs involved fusing the DNA binding domain to the GR element to make it inducible. A similar construct has been used to study Slug function (LaBonne and Bronner-Fraser, 2000). We were aware of the possibility that this construct could affect the function of both genes because of the sequence similarity between Snail and Slug in the zinc-finger region (Manzanares et al., 2002). It is worth noting that we were able to show that in the mesoderm, where there is a complementary expression of Slug and Snail, the inhibition of Slug function by this Slug dominant-negative version could only be rescued by Slug and not by co-injection of Snail (Mayor et al., 2000). This suggests that it specifically works inhibiting Slug function. However, the limitation of this approach is particularly relevant when studying the function of Snail family members in neural crest development in Xenopus, as both genes are co-expressed in this tissue. Therefore, we generated another dominant negative construct that contained the N-terminal region of Snail, which is highly divergent from that of Slug. Using this construct, a similar inhibition of neural crest markers was observed, indicating that the activity of Snail is indeed required for the 
early specification of the neural crest. Furthermore, we were able to rescue the effect of the Snail dominant-negative using Slug co-injection, but we were not able to rescue the effect of Slug dominant-negative by using Snail co-expression. Taken together, these results strongly support the conclusion that Snail is upstream of Slug in the specification of the neural crest cells.

We have also shown that Snail probably functions as a transcriptional repressor, as the injection of a repressor construct produces the same phenotype as that of the wild-type Snail, and has the opposite effect to that of an activator construct. This is not surprising, as Snail family members have been shown to act as repressors in many species from Drosophila to humans (Nieto, 2002). Thus, the simplest interpretation of our data is that Snail represses the expression of a factor that prevents the expression of the other neural crest markers. The ability of Snail to transform animal caps into cells expressing neural crest markers and the inhibition of epidermal markers in the whole embryo suggest that such an unknown factor should be expressed in the ectoderm. Candidate molecules include BMP4 and the genes downstream of it, such as Msxl and the Dlx genes (Bendall and Abate-Shen, 2000). Indeed, the observation that Snail is able to repress BMP4 expression in animal caps supports the idea that this factor could be the target of Snail repression during neural crest specification. A similar function has been attributed to Slug in the dorsal mesoderm, where this gene influences the development of the Spemann organiser by repressing BMP4 expression in this tissue (Mayor et al., 2000). Thus, our results suggest that Snail is also a repressor of BMP4, and that this repression is required for the specification of the neural crest territory. This conclusion is supported by the model in which the neural crest is induced by a gradient of BMP activity (Marchant et al., 1998; Morgan and Sargent, 1997; Nguyen et al., 1998). Thus, if Snail were to repress BMP4, Snail injections will cause the levels of BMP4 to be reduced over a more extended area, thereby displacing the threshold level necessary to specify neural crest to the epidermal region and leading to the conversion of ectodermal cells to neural crest.

\section{Snail is involved in neural crest migration}

As Snail is expressed in migratory neural crest cells, we investigated what function it may fulfill during migration. Because we have shown that Snail interferes with neural crest specification, we adopted the same approach to study the role of Snail in migration by activating the inducible constructs only once neural crest precursors had already been formed. Overexpression of Snail at the midneurula stage produced an increase in the number of migrating neural crest cells. This is in agreement with results obtained by overexpressing Slug in the chick (Del Barrio and Nieto, 2002). Blocking Snail activity with dominant-negative constructs led to a reduction in neural crest migration as has also been described for Slug in Xenopus (LaBonne and Bronner-Fraser, 2000). However, some migratory cells were observed in the manipulated embryos, suggesting that other factors probably cooperate with Snail in the migratory process. Among the possible factors, Slug is a good candidate because it has already been proposed to act as a maintenance factor of the mesenchymal phenotype (Ros et al., 1997). Moreover, it has been suggested that these two factors cooperate during crest migration in the chick and mouse, as a subpopulation of migratory crest express both genes (Sefton et al., 1998; Cano et al., 2000).

\section{Snail and Slug show functional equivalence}

In this work, we show that Snail plays a key role in the development of the neural crest in Xenopus embryos, similar to that seen after Slug overexpression (LaBonne and BronnerFraser, 1998). One explanation for this is that both genes are functionally equivalent and that overexpression experiments cannot discriminate between the two. This possibility was tested by comparing the effect of injecting Snail or Slug mRNAs into Xenopus embryos. No obvious differences could be detected in the effects of either gene on the expression of neural crest markers or the migration of the neural crest. Furthermore, overexpressing Snail or Slug from chick and mouse also produced similar results, supporting earlier data where it was suggested that both genes are functionally equivalent in chick embryos (Del Barrio and Nieto, 2002). This also indicates that overexpression in Xenopus cannot discriminate between their specific activities. Along the same lines, the results obtained with dominant-negative constructs may also reflect a lack of specificity. As mentioned above, to avoid such a problem, we have used a dominant-negative construct, which is specific as it contains the highly divergent region of the different family members that lies outside of the zinc-finger domains (Manzanares et al., 2001) and we were able to rescue the effect of Snail dominant negative by coexpression of Snail wild type. In summary, considering that Snail is expressed before Slug in the crest precursors, that Snail but not Slug is able to induce neural crest markers in animal cap assays and that Snail is able to induce Slug, we believe that Snail may be responsible for at least some of the functions previously associated to Slug. Thus, we propose that Snail rather than Slug plays an early role in neural crest development in Xenopus, as has been suggested in mammals (Sefton et al., 1998; Jiang et al., 1998; Cano et al., 2000; Carver et al., 2001). As mentioned before, Slug may fulfil similar or additional functions in the premigratory population at later stages and also during migration.

\section{The relationship between Snail, Slug and other neural crest markers}

A number of different transcription factors have been shown to be expressed in the prospective neural crest of Xenopus embryos (Meis1, Pbxl, several Zic genes, FoxD3 and Slug), and have been implicated in its early development (Sasai et al., 2001; Nakata et al., 2000; Pohl and Knöchel, 2001; Maeda et al., 2001; Maeda et al., 2002; LaBonne and Bronner-Fraser, 1998; LaBonne and Bronner-Fraser, 2000; Mayor et al., 2000). We now show that Snail fulfils a similar function. We show that Snail is the earliest specific marker of crest precursors and we believe that it lies upstream of these other factors in the genetic cascade of crest specification for the following reasons: (1) Snail is able to induce all the above-mentioned neural crest markers; (2) considering the onset of expression, the Zic genes are expressed in the prospective neural plate from very early stages and become restricted to the neural crest territory long after Snail; (3) Slug and FoxD3 are expressed in the precursors of the neural crest after Snail; (4) all the above-mentioned genes including Zic5 (Nakata et al., 2000), with the exception of Snail and Slug, induce neural plate markers as well as neural 
crest markers when assayed in animal caps; and (5) Slug is incapable of inducing any known crest markers in animal caps.

Furthermore, we have recently proposed a model for neural crest induction (Villanueva et al., 2002; Aybar and Mayor, 2002 ) in which the entire neural plate border is induced first but later crest production is restricted to the posterior regions of the neural fold. This model predicts that the earliest crestspecific genes induced should be expressed in the entire neural plate border, with later genes being restricted to the definitive neural crest-forming region. Interestingly, Snail is the only gene described to date whose expression fits in well with this model. Its early expression along the entire length of the neural plate border correlates with the first phase of crest specification (Fig. 2H). However, definitive crest will be only produced at more posterior levels upon the action of signalling molecules (Wnt, FGF, retinoic acid) which will maintain high levels of Snail expression (Fig. 2I). In agreement with this, FGFR signalling is needed for the maintenance of Snail expression in the mouse primitive streak (Ciruna and Rossant, 2001). This would justify that the low levels of Snail expression observed in the anterior neural plate do not generate crest, and would also explain its ability to induce all neural crest markers with the high dose used in animal cap assays. Finally, Snail is the only gene described to date able to specifically induce all neural crest markers in the absence of neural plate markers. Altogether, our data indicate that Snail lies high and upstream of Slug in the hierarchy of crest specification, being activated very early in the territory competent to become crest.

We thank Y. Sasai, J. Aruga, J. Gurdon, T. Sargent, D. Turner and N. Papalopulu for reagents used in this research. Work in the laboratory of R. M. was supported by an International Research Scholar Award from the Howard Hughes Medical Institute to R. M., and by grants from Fondecyt (\#1020688 to R. M., \#3010061 to M. J. A.), Fundación Antorchas (\#13953-3 to M. J. A.), the Millennium Program (P99-137F to R. M.), the Spanish Ministry of Science and Technology (PM98-125 and BMC2002-00383) to M. A. N. and a collaborative project from CSIC/CONICYT (2001CL0023) to R. M. and M. A. N.

\section{REFERENCES}

Aybar, M. J. and Mayor, R. (2002). Early induction of neural crest cells, lessons learned from frog, fish and chick. Curr. Opin. Genet. Dev. 12, $452-$ 458.

Bendall, A. J. and Abate-Shen, C. (2000). Roles for Msx and Dlx homeoproteins in vertebrate development. Gene 247, 17-31.

Cano, A., Pérez, M. A., Rodrigo, I., Locascio, A., Blanco, M. J., del Barrio, M. G., Portillo, F. and Nieto, M. A. (2000). The transcription factor Snail controls epithelial-mesenchymal transitions by repressing E-cadherin expression. Nat. Cell Biol. 2, 76-83

Carl, T. F., Dufton, C., Hanken, J. and Klymkowsky, M. W. (1999). Inhibition of neural crest migration in Xenopus using antisense slug RNA. Dev. Biol. 213, 101-115.

Carver, E. A., Jiang, R., Lan, Y., Oram, K. F. and Gridley, T. (2001). The mouse Snail gene encodes a key regulator of the epithelial-mesenchymal transition. Mol. Cell. Biol. 21, 8184-8188.

Chomczynski, P. and Sacchi, N. (1987). Single-step method of RNA isolation by acid guanidinium thiocyanate-phenol-chloroform extraction. Anal. Biochem. 162, 156-159.

Ciruna, B. and Rossant, J. (2001). FGF signaling regulates mesoderm cell fate specification and morphogenetic movement at the primitive streak. Dev. Cell 1, 32-47.

Del Barrio, M. G. and Nieto, M. A. (2002). Overexpression of Snail family members highlights their ability to promote chick neural crest formation. Development 129, 1583-1593.

Duband, J. L., Monier, F., Delannet, M. and Newgreen, D. (1995). Epithelium-mesenchyme transition during neural crest development. Acta Anat. 154, 63-78.

Essex, L. J., Mayor, R. and Sargent, M. G. (1993). Expression of Xenopus snail in mesoderm and prospective neural fold ectoderm. Dev. Dyn. 198, 108-122.

García-Castro, M. I., Marcell, C. and Bronner-Fraser, M. (2002). Ectodermal Wnt function as a neural crest inducer. Science 297, 848-851.

Harland, R. M. (1991). In situ hybridization: an improved whole-mount method for Xenopus embryos. Methods Cell Biol. 36, 685-695.

Harland, R. and Weintraub, H. (1985). Translation of mRNA injected into Xenopus oocytes is specifically inhibited by antisense RNA. Cell Biol. 101, 1094-1099.

Harris, W. A. and Hartenstein, V. (1991). Neuronal development without cell division in Xenopus embryos. Neuron 6, 499-516.

Hay, E. D. (1995). An overview of epithelio-mesenchymal transformation. Acta Anat. 154, 8-20.

Hopwood, N. D., Pluck, A. and Gurdon, J. B. (1989). A Xenopus mRNA related to Drosophila twist is expressed in response to induction in the mesoderm and the neural crest. Cell 59, 893-903.

Jiang, R., Lan, Y., Norton, C. R., Sundberg, J. P. and Gridley, T. (1998). The Slug gene is not essential for mesoderm or neural crest development in mice. Dev. Biol. 198, 277-285.

Joanes, E., Sargent, T. D. and Dawid, I. B. (1985). Epidermal keratin gene expressed in embryos of Xenopus laevis. Proc. Natl. Acad. Sci. USA 82, 5413-5417.

Knecht, A. K. and Bronner-Fraser, M. (2002). Induction of the neural crest: a multigene process. Nat. Rev. Genet. 3, 453-461.

Kolm, P. J. and Sive, H. L. (1995). Efficient hormone-inducible protein function in Xenopus laevis. Dev. Biol. 171, 267-272.

LaBonne, C. and Bronner-Fraser, M. (1998). Neural crest induction in Xenopus, evidence for a two-signal model. Development 125, 24032414.

LaBonne, C. and Bronner-Fraser, M. (2000). Snail-related transcriptional repressors are required in Xenopus for both the induction of the neural crest and its subsequent migration. Dev. Biol. 221, 195-205.

Le Douarin, N. M. and Kalcheim, C. (1999). The Neural Crest. Cambridge, UK: Cambridge University Press.

Linker, C., Bronner-Fraser, M. and Mayor, R. (2000). Relationship between gene expression domains of Xsnail, Xslug, and Xtwist and cell movement in the prospective neural crest of Xenopus. Dev. Biol. 224, 215 225

Locascio, A. and Nieto, M. A. (2001). Cell movements during vertebrate development, integrated tissue behaviour versus individual cell migration. Curr. Opin. Genet. Dev. 11, 464-469.

Maeda, R., Mood, K., Jones, T. L., Aruga, J., Buchberg, A. M. and Daar, I. O. (2001) Xmeis1, a protooncogene involved in specifying neural crest cell fate in Xenopus embryos. Oncogene 20, 1329-1342.

Maeda, R., Ishimura, A., Mood, K., Park, E. K., Buchberg, A. M. and Daar, I. O. (2002). Xpbxlb and Xmeislb play a collaborative role in hindbrain and neural crest gene expression in Xenopus embryos. Proc. Natl. Acad. Sci. USA 99, 5448-5453.

Mancilla, A. and Mayor, R. (1996). Neural crest formation in Xenopus laevis, mechanisms of Xslug induction. Dev. Biol. 177, 580-589.

Manzanares, M., Locascio, A. and Nieto, M. A. (2001). The increasing complexity of the Snail superfamily in metazoan evolution. Trends Genet. 17, 178-181.

Marchant, L., Linker, C., Ruiz, P., Guerrero, N. and Mayor, R. (1998). The inductive properties of mesoderm suggest that the neural crest cells are specified by a BMP gradient. Dev. Biol. 198, 319-329.

Mayor, R. and Aybar, M. J. (2001). Induction and development of neural crest in Xenopus laevis. Cell Tissue Res. 305, 203-209.

Mayor, R., Essex, L. J., Bennet, M. F. and Sargent, M. G. (1993). Distinct elements of the Xsna promoter are required for mesodermal and ectodermal expression. Development 119, 661-671.

Mayor, R., Morgan, R. and Sargent, M. G. (1995). Induction of the prospective neural crest of Xenopus. Development 121, 767-777.

Mayor, R., Guerrero, N., Young, R. M., Gomez-Skarmeta, J. L. and Cuellar, C. (2000). A novel function for the Xslug gene, control of dorsal mesendoderm development by repressing BMP-4. Mech. Dev. 97, 47-56.

Meyer, D., Durliat, M., Senan, F., Wolff, M., Andre, M., Houdry, J. and 
Remy, P. (1997). Ets-1 and Ets-2 proto-oncogenes exhibit differential and restricted expression patterns during Xenopus laevis oogenesis and embryogenesis. Int. J. Dev. Biol. 41, 607-620.

Mizuseki, K., Kishi, M., Matsui, M., Nakanishi, S. and Sasai, Y. (1998). Xenopus Zic-related-1 and Sox-2, two factors induced by chordin, have distinct activities in the initiation of neural induction. Development 125, 579-587.

Morgan, R. and Sargent, M. G. (1997). The role in neural patterning of translation initiation factor eIF4AII; induction of neural fold genes. Development 124, 2751-2760.

Nagai, T., Aruga, J., Takada, S., Günther, T., Spörle, R., Schughart, K. and Mikoshiba, K. (1997). The expression of mouse Zic1, Zic2, Zic3 gene suggest an essential role for Zic genes in body pattern formation. Dev. Biol. 182, 299-313.

Nakata, K., Nagai, T., Aruga, J. and Mikoshiba, K. (1997). Xenopus Zic3, a primary regulator both in neural and neural crest development. Proc. Natl. Acad. Sci. USA 94, 11980-11985.

Nakata, K., Nagai, T., Aruga, J. and Mikoshiba, K. (1998). Xenopus Zic family and its role in neural and neural crest development. Mech. Dev. 75, 43-51.

Nakata, K., Koyabu, Y., Aruga, J. and Mikoshiba, K. (2000). A novel member of the Xenopus Zic family, Zic5, mediates neural crest development. Mech. Dev. 99, 83-91.

Nguyen, V. H., Schmid, B., Trout, J., Connors, S. A., Ekker, M. and Mullins, M. C. (1998). Ventral and lateral regions of the zebrafish gastrula, including the neural crest progenitors, are established by a bmp $2 / s w i r l$ pathway of genes. Dev. Biol. 199, 103-110.

Nieto, M. A. (2001). The early steps of neural crest development. Mech. Dev. 105, 27-35.

Nieto, M. A. (2002). The Snail superfamily of zinc finger transcription factors. Nat. Rev. Mol. Cell. Biol. 3, 155-166.
Nieto, M. A., Sargent, M. G., Wilkinson, D. G. and Cooke, J. (1994). Control of cell behavior during vertebrate development by Slug, a zinc finger gene. Science 264, 835-839.

Nieuwkoop, P. D. and Faber, J. (1967). Normal table of Xenopus laevis (Daudin). Amsterdam: North Holland.

Perris, R. and Perissinotto, D. (2000). Role of the extracellular matrix during neural crest cell migration. Mech. Dev. 95, 3-21.

Pohl, B. S. and Knochel, W. (2001). Overexpression of the transcriptional repressor FoxD3 prevents neural crest formation in Xenopus embryos. Mech. Dev. 103, 93-106.

Ros, M., Sefton, M. and Nieto, M. A. (1997). Slug, a zinc finger gene previously implicated in the early patterning of the mesoderm and the neural crest, is also involved in chick limb development. Development 124, 18211829.

Sasai, N., Mizuseki, K. and Sasai, Y. (2001). Requirement of FoxD3-class signaling for neural crest determination in Xenopus. Development 128, 2525-2536.

Sefton, M., Sanchez, S. and Nieto, M. A. (1998). Conserved and divergent roles for members of the Snail family of transcription factors in the chick and mouse embryo. Development 125, 3111-3121.

Sharpe, C. R. and Goldstone, K. (1997). Retinoid receptors promote primary neurogenesis in Xenopus. Development 124, 515-523.

Smith, J. C., Dale, L. and Slack, J. M. W. (1985). Cell lineage of labels and region-specific markers in the analysis of inductive interactions. J. Embryol. Exp. Morphol. 89, 317-331.

Turner, D. L. and Weintraub, H. (1994). Expression of achaete-scute homolog 3 in Xenopus embryos converts ectodermal cells to a neural fate. Genes Dev. 8, 1434-1447.

Villanueva, S., Glavic, A., Ruiz, P. and Mayor, R. (2002). Posteriorization by FGF, Wnt, and Retinoic Acid is required for neural crest induction. Dev. Biol. 241, 289-301. 